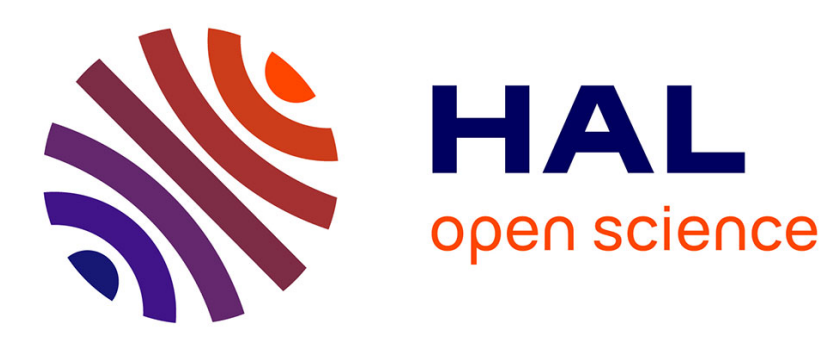

\title{
Dynamics of a cold atom cloud in an anharmonic trap
}

\author{
Andrea Bertoldi, L. Ricci
}

\section{To cite this version:}

Andrea Bertoldi, L. Ricci. Dynamics of a cold atom cloud in an anharmonic trap. Physical Review A : Atomic, molecular, and optical physics [1990-2015], 2010, 81, pp.063415. 10.1103/PhysRevA.81.063415 . hal-00628524

\section{HAL Id: hal-00628524 \\ https://hal-iogs.archives-ouvertes.fr/hal-00628524}

Submitted on 4 Dec 2015

HAL is a multi-disciplinary open access archive for the deposit and dissemination of scientific research documents, whether they are published or not. The documents may come from teaching and research institutions in France or abroad, or from public or private research centers.
L'archive ouverte pluridisciplinaire HAL, est destinée au dépôt et à la diffusion de documents scientifiques de niveau recherche, publiés ou non, émanant des établissements d'enseignement et de recherche français ou étrangers, des laboratoires publics ou privés. 


\title{
Dynamics of a cold atom cloud in an anharmonic trap
}

\author{
Andrea Bertoldi* and Leonardo Ricci \\ Dipartimento di Fisica, Università di Trento, I-38123 Trento-Povo, Italy
}

(Received 8 February 2010; published 17 June 2010)

\begin{abstract}
A combined gravitomagnetic trap was used to measure how the anharmonicity of a trapping potential affects the dynamics of a cold atomic cloud. A displacement of the effective potential minimum as a function of the ensemble temperature was observed. The effect is a direct consequence of the thermal nature of the atomic sample. The results of both a theory approach to the dynamical evolution of the atomic cloud and a numerical simulation are in very good agreement with the experimental observations. The effect can be exploited to devise a novel measurement technique for the temperature of a trapped atomic cloud, as well as to separate thermal and condensed phases in Bose-Einstein condensation experiments.
\end{abstract}

DOI: 10.1103/PhysRevA.81.063415

PACS number(s): 37.10.Gh, 07.55.Db, 85.90.+h

\section{INTRODUCTION}

Trapping [1] and guiding [2,3] neutral cold atoms with static magnetic fields is a well-developed technique, and it represents an important tool for reaching Bose-Einstein condensation [4-6]. Harmonic confining configurations are normally adopted in cold atom experiments. This allows for the use of simple, standard techniques to analyze the dynamics of trapped particles; for example, the equipartition theorem is valid [7]. In contrast, anharmonicity not only brings about a rise in complexity, but also is at the basis of many interesting phenomena and applications. Before the advent of cold atom techniques, it was proved that a particle trapped in an anharmonic potential emits a narrow line with no Doppler broadening [8]. More recently, the anharmonicity of the trap has been exploited to turn laser-induced parametric excitation of atomic motion from a heating source into a robust way to force evaporative cooling, both in far-off-resonance optical traps [9] and in magnetic traps [10]. Anharmonic traps opened the way to fast rotating Bose-Einstein condensate (BEC) studies: in this regime, provided that the interaction energy is weak enough, a giant vortex forms and circular superflow at a supersonic speed occurs [11]. Similar effects are presently under investigation for Fermi gases $[12,13]$.

The study of the dynamics of a BEC in a microfabricated magnetic waveguide [14] revealed several nonlinear effects caused by the anharmonic nature of the potential: high harmonics generation for the motion of the center of mass (CM), coupling between internal and external dynamics, mode mixing, and chaotic dynamics in case of strong excitations. More in general, the scaling of the trap dimensions in miniaturized trapping configurations [15], though allowing for stronger confinement, yields a higher degree of nonlinearity. This issue poses stringent constraints on integrated atom optics aiming at high-precision inertial sensing devices and on the measurement of atom-surface interactions.

In this paper, we study the dynamics of a cold sample of ${ }^{87} \mathrm{Rb}$ after its transfer in an anharmonic trapping potential resulting from the combination of a suitably tailored magnetic field and standard uniform gravity [16]. The analysis, both

\footnotetext{
*Present address: Institut d'Optique, Campus Polytechnique, RD 128, F-91127 Palaiseau, France; andrea.bertoldi@institutoptique.fr
}

theoretical and experimental, shows that the dynamics is strongly affected by anharmonic terms in the potential expansion. Moreover, the equilibrium point of the CM of a cloud depends on the temperature of the loaded atoms. Remarkably, due to the finite initial temperature and extension of the cloud, even an optimal atomic transfer leads to a vertical oscillation of the cloud.

The effect discussed here can be used to devise a novel way to measure the temperature of a cold sample of atoms confined in an anharmonic trap. Moreover, it could play a role in schemes aiming at the spatial separation of the thermal and the condensed phase in BEC experiments.

The article is structured as follows. In Sec. II the role of the anharmonicity of a combined gravitomagnetic trap in the atomic dynamics is theoretically discussed, whereas Sec. III describes the results of a numerical simulation that mimics the ensemble behavior. The experimental setup is the topic of Sec. IV. Section V presents the experimental data, which are in very good agreement with the results of the numerical simulation. Conclusions and perspectives are reported in Sec. VI.

\section{ANHARMONIC TRAPPING POTENTIAL}

In this section the effects of anharmonicity of a combined, cylindrically symmetric gravitomagnetic potential on the dynamics of a trapped atomic cloud are analyzed. The general gravitomagnetic potential for a paramagnetic atom having magnetic moment $\mu$ is [16]

$$
U_{\mathrm{GM}}=U_{\mathrm{G} 0}+m g z+\mu|\boldsymbol{B}|,
$$

where $U_{\mathrm{G} 0}$ represents an offset value, $m$ the atom mass, and $g$ the modulus of the gravity acceleration. The uniform gravitational field is assumed to be directed along $z$, which is chosen to be the symmetry axis of the applied magnetic field and the related cylindrical coordinate system $(\rho, \phi, z)$.

Let a point on the $z$ axis be the origin. The Taylor expansion of the magnetic field in a neighborhood of the origin is determined up to the third order by the four parameters $B_{0}, B^{\prime}$, $B^{\prime \prime}$, and $B^{\prime \prime \prime}$, which correspond to the first four axial magnetic field moments, evaluated in the origin: the axial bias field $B_{z}$, 
the axial gradient of the axial component of the magnetic field $\partial B_{z} / \partial z$, and so on. The magnetic field components result:

$$
\begin{aligned}
B_{\rho}= & -\frac{1}{2} B^{\prime} \rho-\frac{1}{2} B^{\prime \prime} \rho z \\
& +-\frac{1}{4} B^{\prime \prime \prime}\left(z^{2}-\frac{1}{4} \rho^{2}\right) \rho+O\left(r^{4}\right), \\
B_{z}= & B_{0}+B^{\prime} z+\frac{1}{2} B^{\prime \prime}\left(z^{2}-\frac{\rho^{2}}{2}\right) \\
& +\frac{1}{2} B^{\prime \prime \prime}\left(\frac{1}{3} z^{3}-\frac{1}{2} \rho^{2} z\right)+O\left(r^{4}\right),
\end{aligned}
$$

where $O\left(r^{4}\right)$, with $r^{2}=\rho^{2}+z^{2}$, groups terms of order higher than 3. If $B_{0}$ is different from 0 and the $z$ axis is oriented so that $B_{0}>0$, the Taylor expansion of the modulus of the magnetic field in a suitable neighborhood of the origin and up to the second order results in

$$
|\boldsymbol{B}|=B_{0}+B^{\prime} z+\frac{1}{2} B^{\prime \prime} z^{2}+\frac{1}{2} \chi B^{\prime \prime} \rho^{2}+O\left(r^{3}\right),
$$

where $\chi$ is the ratio between the radial and the axial curvature:

$$
\chi \equiv \frac{1}{4 B^{\prime \prime}}\left(\frac{B^{\prime 2}}{B_{0}}-2 B^{\prime \prime}\right)
$$

Setting an axial point to be the minimum of the combined potential requires the gradient of $U_{\mathrm{GM}}$ to vanish there and the Hessian of $U_{\mathrm{GM}}$ to be non-negative in its neighborhood. With regard to the radial gradient, the condition is always satisfied if $\rho=0$. The following discussion is devoted to this axial solution only. However, as we aim at investigating the dynamics of an atom cloud rather than a single atom, we consider the axial gradient condition in a neighborhood of the axis, thus allowing $\rho$ to be (slightly) different from 0 . Differentiating along $z$ and equating to 0 , Eq. (1) gives

$$
\gamma+\frac{\boldsymbol{B} \cdot \partial_{z} \boldsymbol{B}}{|\boldsymbol{B}|}=0
$$

where $\gamma \equiv m g / \mu$ is the magnetic field gradient that compensates gravity. For the $F=2, m_{F}=2$ state of ${ }^{87} \mathrm{Rb}, \gamma=$ $15.4 \mathrm{G} / \mathrm{cm}$. This value has to be doubled for atoms either in the $F=2, m_{F}=1$ state or in the $F=1, m_{F}=-1$ state. Let $B^{\prime}=-\gamma$. Then, assuming again that $B_{0}>0$, using Eqs. (2) and (3), and keeping the terms up to the second order, Eq. (5) becomes

$$
(1+\epsilon) z^{2}-2 \delta z-\frac{1}{2} \chi \rho^{2}\left(1+\frac{\epsilon}{\chi}\right)=0,
$$

where the lengthlike parameter $\delta$ is defined as $\delta \equiv-B_{0} / 2 B^{\prime}$, whereas the dimensionless parameter $\epsilon$, defined as $\epsilon \equiv$ $B_{0} B^{\prime \prime \prime} / 2 B^{\prime} B^{\prime \prime}$, expresses the entity of the third-order component $B^{\prime \prime \prime}$ in terms of the three lower-order ones. On the axis $(\rho=0)$, one of the two solutions for the axial position of the trap center coincides - as expected — with the origin. Off axis, the 0 of Eq. (6) next to the origin results in

$$
z_{0}=\frac{\delta-\sqrt{\delta^{2}+\frac{1}{2} \chi \rho^{2}(1+\epsilon)\left(1+\frac{\epsilon}{\chi}\right)}}{1+\epsilon} .
$$

According to Eq. (7), and as a consequence of the nonlinearity of the potential, the axial position of the trap bottom progressively drops with $\rho$. The nonlinearity is mainly due
TABLE I. Main four terms in the Taylor expansion of the axial magnetic field in the configuration center and related values of significant parameters discussed in Sec. II.

\begin{tabular}{lc}
\hline \hline Parameter & Value \\
\hline$B_{0}(\mathrm{G})$ & 0.50 \\
$B^{\prime}\left(\mathrm{G} \mathrm{cm}^{-1}\right)$ & -15.4 \\
$B^{\prime \prime}\left(\mathrm{G} \mathrm{cm}^{-2}\right)$ & 91 \\
$B^{\prime \prime \prime}\left(\mathrm{G} \mathrm{cm}^{-3}\right)$ & 25 \\
$\chi$ & 0.80 \\
$\delta(\mathrm{mm})$ & 0.16 \\
$\epsilon$ & $-4.5 \times 10^{-3}$ \\
$T_{0}(\mu \mathrm{K})$ & 3.2 \\
\hline \hline
\end{tabular}

to the square-root operation required by the modulus of the field $|\boldsymbol{B}|$. It is important to remark that this occurs even in the case of vanishing $B^{\prime \prime \prime}$ and despite the apparent harmonicity of the potential resulting from Eq. (3). If $\epsilon \ll 1$, as in the experimental implementation described later (see Table I), Eq. (7) can be written as

$$
z_{0}=\delta-\sqrt{\delta^{2}+\frac{1}{2} \chi \rho^{2}} .
$$

Henceforth, we consider the atom cloud confined in the combined trap as an ensemble and apply the ergodic hypothesis [7]; related statistical averages are denoted " \langle\rangle ." Averaging Eq. (8) requires knowledge of the distribution of $\rho$. This can be evaluated by assuming the trap to be harmonic in first approximation, so that the spatial distribution of atoms in each Cartesian direction is Gaussian. It follows that

$$
P(\rho)=\frac{2 \rho}{\left\langle\rho^{2}\right\rangle} e^{-\rho^{2} /\left\langle\rho^{2}\right\rangle} .
$$

The average of $z_{0}(\rho)$, given by Eq. (8), over the distribution given by Eq. (9) results in

$$
\left\langle z_{0}\right\rangle=-\delta \frac{\sqrt{\pi}}{2} \frac{e^{a}}{\sqrt{a}} \operatorname{erfc}(\sqrt{a}),
$$

where erfc is the complementary error function and the parameter $a$ is defined as

$$
a \equiv \frac{2 \delta^{2}}{\chi\left\langle\rho^{2}\right\rangle} .
$$

The average radial quadratic displacement $\left\langle\rho^{2}\right\rangle$ can be evaluated in first approximation by using the energy equipartition - the potential energy is then equally divided between the three spatial modes-and the virial theorem. So

$$
\frac{1}{2} \mu_{B} \chi B^{\prime \prime}\left\langle\rho^{2}\right\rangle=\frac{E}{3},
$$

where $E$ is the average total energy. In contrast, the average total energy $E$ of a trapped atom can be evaluated by knowing the temperature $T$ and the spatial distribution of the cloud just before the transfer to the gravitomagnetic trap. The abrupt switching of the confining potential provides a potential energy contribution to the cloud, whereas it does not increase the kinetic energy. It follows that

$$
E=\frac{3}{2} k_{B}\left(T+T_{U}\right),
$$


where $T_{U}$ is a positive, temperature-like parameter accounting for the potential energy contribution.

The combination of Eqs. (10)-(13) yields the following dependence of the CM equilibrium position $\left\langle z_{0}\right\rangle$ on the initial temperature $T\left(a=\frac{T_{0}}{T+T_{U}}\right)$ :

$$
\left\langle z_{0}\right\rangle=-\delta \frac{\sqrt{\pi}}{2} e^{\frac{T_{0}}{T+T_{U}}} \sqrt{\frac{T+T_{U}}{T_{0}}} \operatorname{erfc}\left(\sqrt{\frac{T_{0}}{T+T_{U}}}\right),
$$

where $T_{0}$, defined as

$$
T_{0} \equiv \frac{\mu_{B}}{k_{B}} \frac{B_{0}^{2} B^{\prime \prime}}{2 B^{\prime 2}},
$$

is a second temperature-like parameter depending exclusively on the characteristics of the trapping magnetic field.

Equation (14) predicts the curve representing $\left\langle z_{0}\right\rangle$ vs. $T$-and vs. $T_{U}$ - to be decreasing. Remarkably, even considering the transfer of a pointlike atom cloud prepared at the trap minimum $\left(T_{U}=0\right.$, corresponding to an optimal mode matching), the cloud then has to vertically oscillate with an amplitude proportional to the temperature. At very low temperatures $\left(T \ll T_{0}\right),\left\langle z_{0}\right\rangle$ linearly decreases with $T$ as $d\left\langle z_{0}\right\rangle / d T \simeq-\delta / 2 T_{0}$, independently of $T_{U}$. All these behaviors are confirmed by the numerical simulation described in the next section.

\section{NUMERICAL SIMULATION}

To study the atomic cloud dynamics in a complete nonlinear regime, we carried out a numerical simulation by integrating the dynamic equations of an initial set of atoms. The simulation uses a fourth-order Runge-Kutta algorithm [17] to evaluate the individual trajectory of each atom within the combined gravitomagnetic potential. The initial conditions for the simulation are the position and velocity of an ensemble of atoms. The coordinates are randomly chosen in such a way that the spatial distribution is Gaussian, spherical, and centered at the origin; the velocity distribution corresponds to a Maxwell-Boltzmann function at a given temperature $T$. Because of the low atomic density observed in the combined gravitomagnetic trap, interparticle collisions are not taken into account. The loss channels represented by collisions with the background gas and by Majorana spin-flips could be easily implemented in the simulation, though without changing the results. The magnetic field adopted in all the simulations shown hereafter is that used in the experiment described in the next paragraph (see Table I).

Two trapped clouds were simultaneously evaluated over a time span of $2 \mathrm{~s}$ after the loading: the first one, henceforth referred to as the upper cloud, represented a set of 1000 atoms in the $F=2, m_{F}=2$ state of ${ }^{87} \mathrm{Rb}$; the second one-henceforth, the lower cloud-represented a set of 1000 atoms either in the $F=2, m_{F}=1$ state or in the $F=1, m_{F}=$ -1 one. For each cloud, the steady-state CM equilibrium position $\left\langle z_{0}\right\rangle$ is obtained by averaging the axial position of all the particles during the evolution interval considered. An alternative possibility is to evaluate the fast Fourier transform of the CM axial evolution and use the DC component; for long enough intervals, the two approaches give similar results.
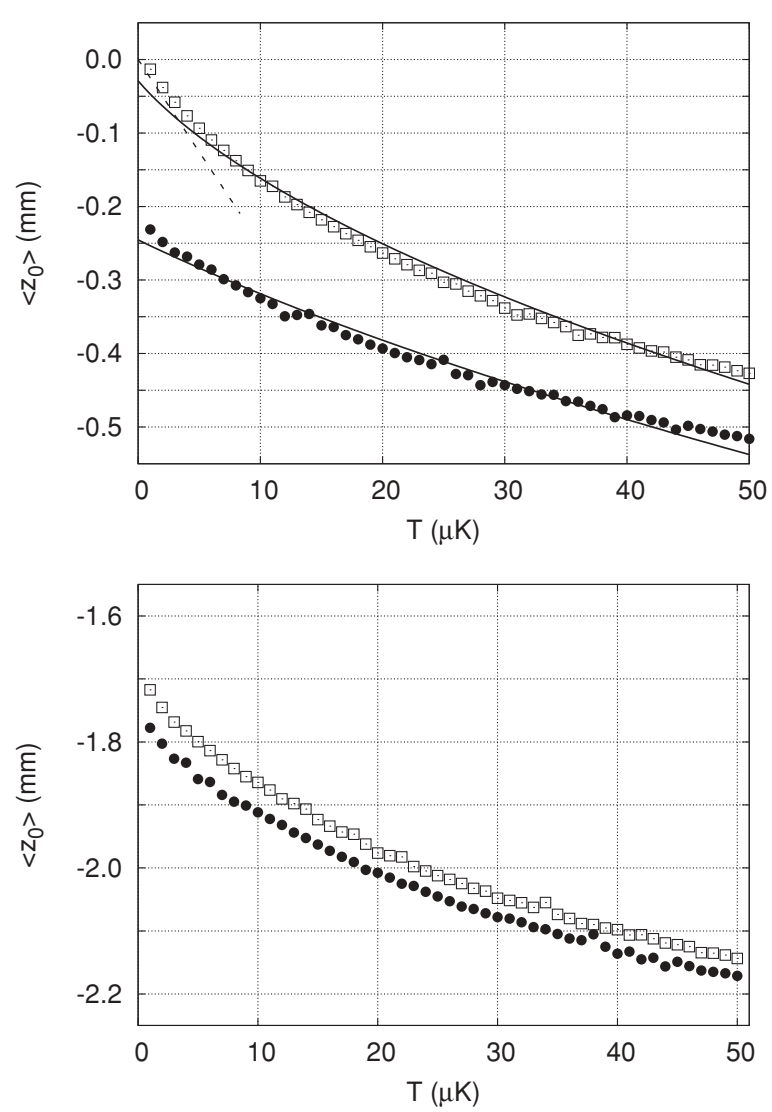

FIG. 1. Center-of-mass equilibrium position $\left\langle z_{0}\right\rangle$ as a function of the initial temperature for the upper (top) and lower (bottom) cloud. The atomic sample is released at $z=0$, with an initial rms radius set to $0.1 \mathrm{~mm}$ (open squares) and $1 \mathrm{~mm}$ (filled circles). The solid lines in the upper plot represent the fit of Eq. (14) to the points, using $T_{U}$ as a unique fit parameter. The dashed line represents the linear behavior at low $T$, having a slope of $-\delta / 2 T_{0}=-0.025 \mathrm{~mm} / \mu \mathrm{K}$. For the lower cloud the oscillation center in the harmonic case occurs at $z=-1.7 \mathrm{~mm}$.

First, the CM equilibrium position $\left\langle z_{0}\right\rangle$ was considered as a function of the temperature $T$ of the transferred ensemble. As shown in Fig. 1, $\left\langle z_{0}\right\rangle$ decreases when the temperature $T$ increases for both the upper cloud and the lower one. Moreover, the outcomes of simulations carried out for initial rms radius $\sqrt{\left\langle\rho^{2}\right\rangle}=0.1$ and $1 \mathrm{~mm}$ show that $\left\langle z_{0}\right\rangle$ is further lowered when the starting size, and thus the initial potential energy $T_{U}$, is increased. The trend predicted by Eq. (14)—shown in Fig. 1 for the upper cloud only-is well confirmed by the simulations.

With regard to the upper cloud, Fig. 2 shows the dependence of $\left\langle z_{0}\right\rangle$ both on the initial rms dimension of the cloud and on its initial axial displacement ( $T$ is set to $5 \mu \mathrm{K}$ ). The behavior resulting from the simulations is well described by Eq. (14) if each of these two parameters does not exceed $1 \mathrm{~mm}$. Outside this range, two approximations used to derive Eq. (14) are no longer valid, so that this equation becomes progressively inadequate to describe the dynamics of the system: first, higher-order terms in the Taylor expansion of the modulus of the magnetic field in Eq. (3) cannot be neglected anymore; second, Eq. (14) relies on the expressions of the energy equipartition and the virial theorem for a harmonic potential, an 

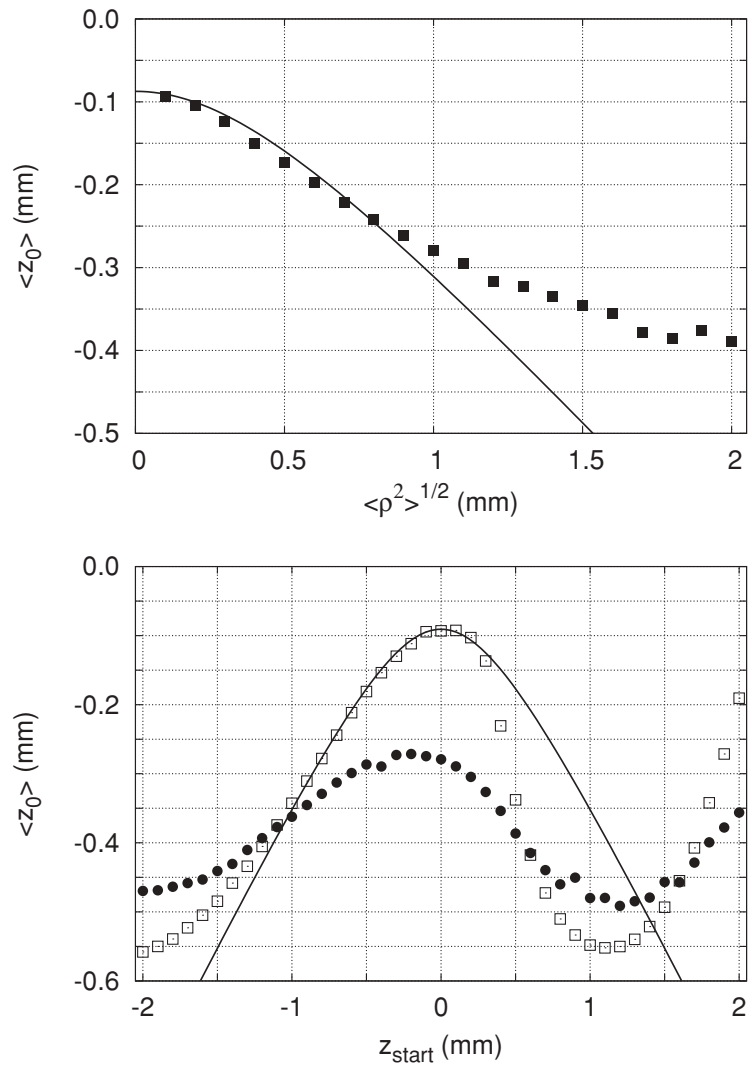

FIG. 2. Center-of-mass equilibrium position $\left\langle z_{0}\right\rangle$ of the upper cloud as a function of the rms radius $\sqrt{\left\langle\rho^{2}\right\rangle}$ (top) and the initial axial position $z_{\text {start }}$ (bottom) of the loaded atomic cloud. In the first case, the solid line represents the behavior predicted by Eq. (14) when the temperature of the transferred cloud was set to $5 \mu \mathrm{K}$. With regard to the second case, open squares and filled circles correspond to the results of two simulations carried out by fixing $\sqrt{\left\langle\rho^{2}\right\rangle}$ to 0.1 and $1 \mathrm{~mm}$, respectively; the solid line represents the behavior for the smaller cloud predicted by Eq. (14) —again, when the temperature of the transferred cloud was set to $5 \mu \mathrm{K}$.

assumption that loses its validity when anharmonicity becomes significant. The marked asymmetric dependence of $\left\langle z_{0}\right\rangle$ on the initial axial position $z_{\text {start }}$ reflects the strong asymmetry of the anharmonicity along the $z$ axis, mainly due to the presence of a 0 of the magnetic field on the axis at $z \cong 0.36 \mathrm{~mm}$. At this point, whose position can be evaluated by solving the third-degree equation obtained by setting Eq. (2b) to 0 , the magnetic force, proportional to the spatial derivative of the magnetic field modulus, is discontinuous.

\section{EXPERIMENTAL APPARATUS}

Our experiment uses a gravitomagnetic trap for ${ }^{87} \mathrm{Rb}$, as described in [18]. A magneto-optical trap (MOT) with typically $10^{6}$ atoms is the source for loading the combined trapping potential. The MOT center is coincident with the minimum of the combined trap to ease the atomic transfer. The magnet configuration is made up of three pairs of coaxial, circular coils. Each pair is used to control one of the first three terms in the Taylor expansion of the magnetic field in the configuration center: in more detail, the internal, central, and external coils mainly act on $B^{\prime \prime}, B^{\prime}$, and $B_{0}$, respectively.
The values adopted in the experiment for these terms, as well as for $B^{\prime \prime \prime}$, are reported in Table I. Typically, a few tens of thousands of atoms are loaded in the combined potential.

The central coil pair is used in the MOT phase to produce a quadrupolar magnetic field having an axial gradient of $-15.4 \mathrm{G} / \mathrm{cm}$ at the origin. This value exactly compensates for the gravitational force on the rubidium atoms in the $F=2$, $m_{F}=2$ state.

The temperature $T$ of the atoms to be loaded in the combined potential was set through a subDoppler cooling phase: by changing the final frequency of the cooling radiation, the temperature could be in the interval from 5 to $40 \mu \mathrm{K}$ with a precision of about $1 \mu \mathrm{K}$. The temperature of the atoms released from the MOT was determined by measuring the ballistic expansion of the freely falling cloud upon turning off the magneto-optical confinement.

Two power MOSFETs are used to fast-switch the magnetic field configuration between MOT (current only in the gradient coils) and the gravitomagnetic trap (same current in the bias, gradient, and curvature coils): the switching interval was measured to be less than $200 \mu$ s. The magnetic field bias could be varied by acting on a variable shunt resistance. A spurious, off-axis component in the origin was compensated by means of a coaxial coil pair with horizontal axis. The coil pair was fed in series to the rest of the magnetic field sources. To optimize the atomic transfer from the MOT to the combined potential (in terms of both the number and the temperature of loaded atoms), the external magnetic field was minimized below $50 \mathrm{mG}$ using three orthogonal couples of coils in Helmholtz configuration.

\section{MEASUREMENTS}

To study the atom dynamics within the combined potential, the region was imaged after a trapping time interval $\Delta t$ varying from 30 to $300 \mathrm{~ms}$, with a $10-\mathrm{ms}$ step. The imaging was carried out with a 2-ms pulse of MOT beams, while the magnetic field was turned off to avoid accelerating the atoms. The fluorescence of the trapped atoms was captured by a high-resolution CCD camera. As expected, two atom clouds were trapped in the gravitomagnetic potential, as shown in the sequence in Fig. 3. The upper cloud is made of atoms in the $F=2, m_{F}=2$ state, whereas the lower one is populated by atoms either in the $F=2, m_{F}=1$ state or in the $F=1$, $m_{F}=-1$ one. The relative population in the two clouds could be controlled by optical pumping. In the measurements presented here, each cloud contains about $2 \times 10^{4}$ atoms. The lifetime of the atoms in the trap was $\tau=4.5(2) \mathrm{s}$.

The left column in Fig. 3 shows an experimental sequence obtained by setting the temperature of the MOT cloud at $5 \mu \mathrm{K}$; images shown were taken at multiples of $50 \mathrm{~ms}$. The right column shows a numerical simulation carried out by assuming the same starting temperature and the same rms radius $(0.2 \mathrm{~mm})$. There is a very good agreement between the experimental data and the numerical data, for both the motion of the $\mathrm{CM}$ and that relative to it. As far as the lower cloud is considered, the agreement can be visually appreciated regarding the vertical oscillation phase, the radial expansion of the ensemble, and the recontraction of the cloud 

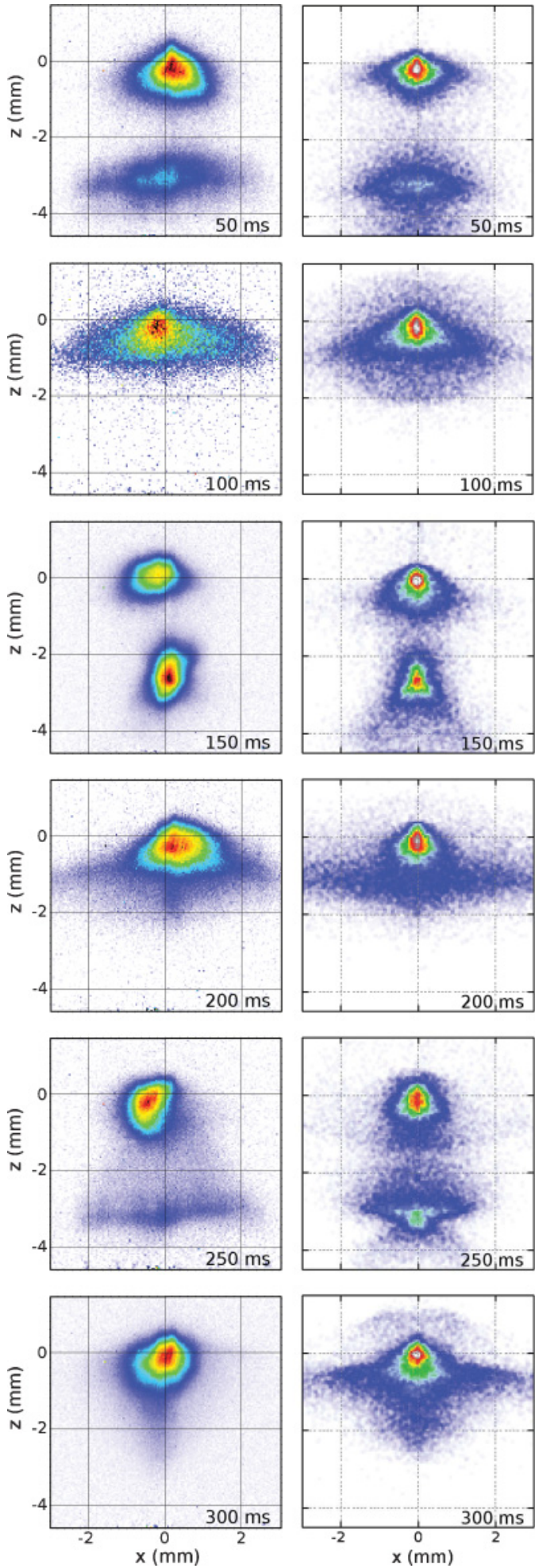

FIG. 3. (Color online) Oscillation of the two clouds within the gravitomagnetic potential: measurement (left) and numerical simulation (right). The temperature and the rms radius of the released atomic ensemble were $5 \mu \mathrm{K}$ and $0.2 \mathrm{~mm}$, respectively. The simulation was run with $2 \times 10^{4}$ atoms in each cloud. The grid spacing is $2 \mathrm{~mm}$.

to a size comparable to the initial one at $z=-2.5 \mathrm{~mm}$ and $\Delta t=150 \mathrm{~ms}$. With regard to the upper cloud, the agreement was verified with a fitting procedure, as discussed here. It must

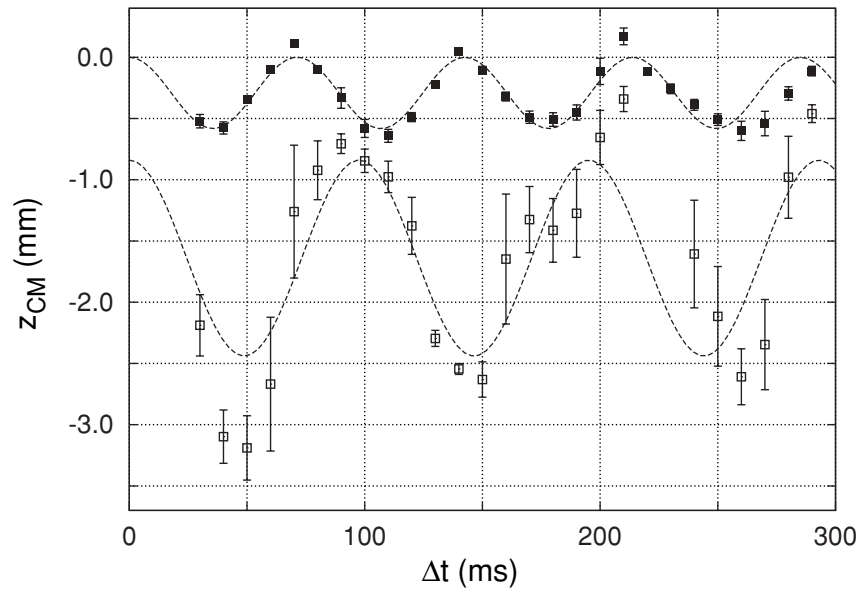

FIG. 4. Center-of-mass time evolution of the two clouds ( $T=$ $12 \mu \mathrm{K})$.

be noted that in the experimental sequence in Fig. 3 the upper cloud shows a horizontal oscillation, due to an out-of-axis initial position. The observed oscillations and breathings of the clouds can be explained in terms of kinematic evolution of the noninteracting particles within the anharmonic potential.

Figure 4 shows the $\mathrm{CM}$ position $z_{\mathrm{CM}}$ of the clouds in an experimental sequence like that shown in Fig. 3. Given a frame, the position was evaluated by fitting the image with a two-dimensional, asymmetric Gaussian distribution. The frequencies resulting from fitting a sinusoidal law of motion for the upper and the lower cloud are 14.0(1) and 10.2(2) Hz, respectively. According to the sinusoidal fit, the lower cloud-as well as the upper one-reaches its maximum at approximately $\Delta t=0$; however, the starting position extrapolated by the fit does not coincide with the position where the MOT is released. This effect is due to the wide axial excursion of the lower cloud, whose outer tail is lost because of contact with the cell windows during the first cycle. The effect becomes worse as the temperature increases, thus preventing the assessment of

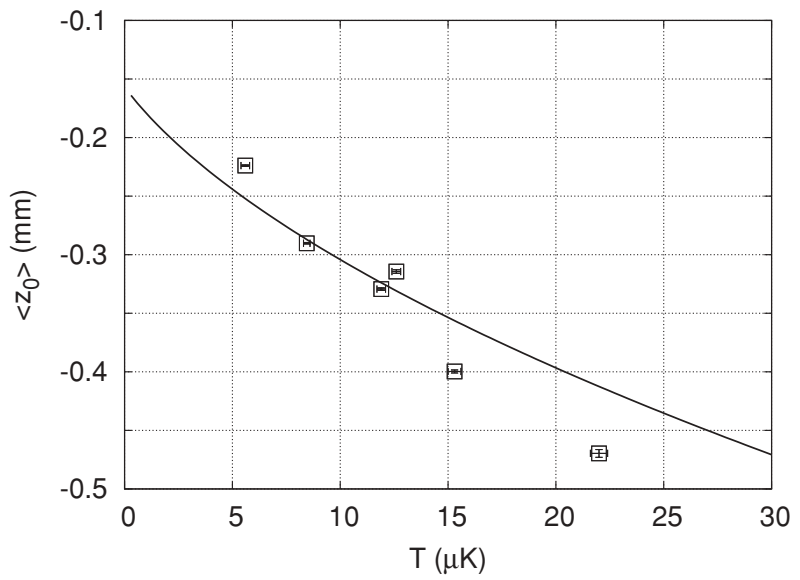

FIG. 5. Axial oscillation center of the upper atomic ensemble in the combined potential (open squares). The solid line represents Eq. (14) "displaced" by a fixed value $\Delta z=-0.16(1) \mathrm{mm}$. This parameter was obtained by fitting on the experimental data the sum of $\Delta z$ and the curve of Eq. (14). The existence of such a displacement is justified by possibly imperfect calibrations of the experimental setup. 
the temperature dependence of the CM equilibrium position for the lower cloud. For this reason, in the following, only the upper cloud is considered.

Effects of the anharmonicity, for example, in terms of coupling between the CM modes and the internal modes of each cloud, are not immediately evident in the time evolution shown in both Fig. 3 and Fig. 4; this is mainly due to the small number of oscillation periods occurring within the reported 300-ms interval. However, anharmonicity effects become manifest when the position of the oscillation center, that is, the measured value of the CM equilibrium position $\left\langle z_{0}\right\rangle$, is considered. This parameter is assessed via the sinusoidal fit in Fig. 4. For the upper cloud, measurement of the dependence of the CM equilibrium position $\left\langle z_{0}\right\rangle$ on the initial temperature $T$ was carried out by analyzing time sequences acquired at different temperatures, ranging from 5 to $22 \mu \mathrm{K}$. Each sequence was made up of 28 images (from 30 to $300 \mathrm{~ms}$, with a $10-\mathrm{ms}$ step) and required about $4 \mathrm{~min}$. The corresponding temperature $T$ was measured before and after the sequence; the resulting temperature variation was typically $0.5 \mu \mathrm{K}$, and a linear drift was assumed. The rms radius of the loaded cloud was typically $0.2 \mathrm{~mm}$.

Figure 5 shows the measured dependence of the CM oscillation center as a function of temperature: the anharmonicity- driven height drop is confirmed, and the agreement with the predictions of Eq. (14) is very good.

\section{CONCLUSIONS}

We have studied the dynamics of a cold atomic cloud loaded in an anharmonic trapping potential. As a consequence of the anharmonicity, the ensemble shows an axial oscillation of the $\mathrm{CM}$ whatever its initial position. The oscillation center shows a temperature-dependent displacement that can be described by a theoretical model relying on the third-order expansion of the magnetic field. The effect was studied by means of a numerical simulation and experimentally verified. The effect could be used to measure the temperature of an atomic cloud without turning off the potential, as well as to separate the thermal and condensed phases in BEC experiments.

\section{ACKNOWLEDGMENTS}

This work was partially supported by the INFM Progetto di Ricerca Avanzata "Photon Matter". A.B. acknowledges support from the EU under IEF Grant No. PIEF-GA-2009235375 .
[1] A. L. Migdall, J. V. Prodan, W. D. Phillips, T. H. Bergeman, and H. J. Metcalf, Phys. Rev. Lett. 54, 2596 (1985).

[2] J. Schmiedmayer, Phys. Rev. A 52, R13 (1995).

[3] B. Ghaffari, J. M. Gerton, W. I. McAlexander, K. E. Strecker, D. M. Homan, and R. G. Hulet, Phys. Rev. A 60, 3878 (1999).

[4] M. H. Anderson, J. R. Ensher, M. R. Matthews, C. E. Wieman, and E. A. Cornell, Science 269, 198 (1995).

[5] C. C. Bradley, C. A. Sackett, J. J. Tollett, and R. G. Hulet, Phys. Rev. Lett. 75, 1687 (1995).

[6] K. B. Davis, M. O. Mewes, M. R. Andrews, N. J. van Druten, D. S. Durfee, D. M. Kurn, and W. Ketterle, Phys. Rev. Lett. 75, 3969 (1995).

[7] L. D. Landau and E. M. Lifshitz, Statistical Physics, 3rd ed., Part 1 (Course of Theoretical Physics, Vol. 5) (ButterworthHeinemann, Oxford, 1980).

[8] Y. V. Baklanov, V. P. Chebotayev, and Y. A. Titov, Appl. Phys. 20, 361 (1979)

[9] N. Poli, R. J. Brecha, G. Roati, and G. Modugno, Phys. Rev. A 65, 021401(R) (2002).
[10] M. Kumakura, Y. Shirahata, Y. Takasu, Y. Takahashi, and T. Yabuzaki, Phys. Rev. A 68, 021401(R) (2003).

[11] K. Kasamatsu, M. Tsubota, and M. Ueda, Phys. Rev. A 66, 053606 (2002).

[12] E. Lundh, New J. Phys. 8, 304 (2006).

[13] K. Howe, A. R. P. Lima, and A. Pelster, Eur. Phys. J. D 54, 667 (2009).

[14] H. Ott, J. Fortágh, S. Kraft, A. Günther, D. Komma, and C. Zimmermann, Phys. Rev. Lett. 91, 040402 (2003).

[15] J. Fortágh and C. Zimmermann, Rev. Mod. Phys. 79, 235 (2007).

[16] L. Ricci, D. Bassi, and A. Bertoldi, Phys. Rev. A 76, 023428 (2007).

[17] W. H. Press, S. A. Teukolsky, W. T. Vetterling, and B. P. Flannery, Numerical Recipes in C (Cambridge University Press, Cambridge, 1992).

[18] A. Bertoldi and L. Ricci, J. Phys. B 41, 155301 (2008). 